\title{
The influence of dipper vs. non-dipper pattern on left ventricular function and ascending aorta dimensions in hypertensive patients in Angola
}

\author{
Ana Feijão', Savarino Victória Pereira², Lorette Cardona', Humberto Morais ${ }^{3}$ \\ 'Departamento de Cardiologia, Luanda Medical Center, Luanda, República de Angola \\ ${ }^{2}$ Serviço de Cardiologia, Hospital Américo Boavida, Luanda, República de Angola \\ ${ }^{3}$ Departamento de Cardiologia, Hospital Militar Principal, Luanda, República de Angola
}

\begin{abstract}
Background. The objective of the present study is to evaluate the effect of the dipper $v$ s. non-dipper patterns on left ventricular function and the dimensions of the ascending aorta in hypertensive patients in Angola.

Material and methods. This is a descriptive, observational study performed at the Department of Cardiology of Luanda Medical Center. For this purpose, information on demographic data, echocardiographic parameters and 24-hour-ambulatory blood pressure measurement parameters was collected. All patients underwent both echocardiographic study and 24-hour-ambulatory blood pressure measurement. Dipper pattern is considered when there is a decrease in the nocturnal $\mathrm{BP}$ in relation to the daytime $\mathrm{BP}$ greater than $10 \%$; if this decrease is not observed either in the systolic blood pressure or in the diastolic blood pressure, the patients are considered non-dippers.

Results. We enrolled 159 patients, of which $85(53.46 \%)$ were dippers and 74 (46.54\%) were non-dippers, and 87 $(54.71 \%)$ were male. The mean age $(\mathrm{SD})$ was $43.62( \pm 10.73)$ years. The patients with the non-dipper pattern had larger ascending aortic diameters $(29.68 \pm 4.88$ vs. $27.8 \pm 5.18, \mathrm{p}=0.001)$, higher left ventricular diastolic diameters $(52.85 \pm 9.24$ vs. $50.24 \pm 4.16, \mathrm{p}=0.027)$ and greater posterior wall thickness $(10.73 \pm 1.94$ vs. $10.07 \pm 1.70$, $\mathrm{p}=0.024)$. In addition, the left ventricular diastolic dysfunction (LVDD) $(B=0.103, p=0.001)$ and the posterior wall $(\mathrm{PW})$ thickness $(B=0.350, \mathrm{p}=0.001)$ are significant estimates of non-dipper pattern.

Conclusions. The results show that hypertensive patients with non-dipper pattern have more alterations in left ventricular geometry, but not in left ventricular systolic and diastolic function.

Key words: essential hypertension; echocardiography; ambulatory blood pressure measurement; dipper, non-dipper




\section{Introduction}

Systemic arterial hypertension (HBP) is a public health problem in Angola, where its prevalence is $23 \%$ [1]. The progression of the disease is strongly associated with functional and structural cardiac alterations, which can be diagnosed by transthoracic echocardiography and, if present, they are indicators of morbidity, mortality and target organ damage. Echocardiography is an extremely useful technique for the evaluation of hypertension and its complications, especially ventricular remodeling and progression to hypertensive heart disease and heart failure, and plays a fundamental role in the diagnosis and therapeutic orientation of these complications [2].

Ambulatory blood pressure measurement (ABPM) is a diagnostic tool that is increasingly used in clinical practice in the evaluation of hypertensive patients [3]. The non-dipper pattern, defined as the insufficient reduction of arterial pressures at night $(<10 \%)$, has been related to a higher cardiovascular and cerebrovascular risk and higher morbidity and mortality with a high prognostic value [4]. However, data about the influence of the non-dipper pattern on cardiac function, when compared to the dipper, in hypertensive patients are conflicting $[5,6]$.

The aim of the present study is to evaluate the effect of the dipper $v$ s. non-dipper pattern on left ventricular function and ascending aorta dimensions in hypertensive patients in Angola.

\section{Material and methods}

\section{Patients studied}

A descriptive observational study was conducted in the Department of Cardiology of Luanda Medical Center in the period from February 2016 to August 2017. We enrolled all hypertensive patients with more than one year of evolution who underwent both transthoracic echocardiography (TTE) and ambulatory blood pressure measurement (ABPM) at the request of the attending physicians.

\section{Echocardiography}

Transthoracic echocardiography (TTE) and the Doppler study were performed with subjects in partial left decubitus position using ultrasound machine (Samsung Medison EKO 7), equipped with a P4-12 transducer. Al studies were performed according to the guidelines of the American Society of Echocardiography [7, 8]. The left atrial area was measured in 2 and 4-apical chamber view. The aortic root diameter, the interventricular septum and the posterior wall thickness, the LV end-diastolic and end-systolic diameter were measured from two-dimensional guided M-mode tracing. The left ventricular ejection fraction (LVEF) was calculated by the Simpson method. Left ventricular filling was assessed by recording mitral flow by standard pulse Doppler technique, and the following parameters were considered: early diastolic peak flow velocity $(\mathrm{E})$, late diastolic peak flow velocity (A) and the ratio of the early to late flow velocity peaks (E/A ratio). Pulsed-wave tissue Doppler imaging was used to measure early diastolic peak wave velocity (e'), and late diastolic peak velocity wave (a'). In addition, the ratio of the early to late wave velocity peaks $\left(e^{\prime} / a^{\prime}\right)$, and ratio of the early diastolic peak flow velocity to early diastolic peak wave velocity (E/e') ratio were calculated.

\section{Ambulatory blood pressure measurement}

The 24-hour ambulatory blood pressure measurement was carried out using a Welch Allyn ABPM 7100 device; the dataset was transferred and analyzed on the CardioPerfect 1.6.6 workstation. The cuff was placed on the non-dominant arm, the patients were asked to perform their usual day-today activities without any restrictions. The patients were instructed to record in the diary the time they went to sleep and they woke up. The device was set to obtain BP readings at 20 min intervals during the daytime period (7:00-23:00 hours) and at $30 \mathrm{~min}$ intervals during the night (23:00-7:00 hours). Each ABPM dataset was first automatically scanned to remove artefactual readings, according to preselected editing criteria. Systolic readings $>$ $260 \mathrm{~mm} \mathrm{Hg}$ or $<70 \mathrm{~mm} \mathrm{Hg}$ and diastolic readings $>150 \mathrm{~mm} \mathrm{Hg}$ or $<40 \mathrm{~mm} \mathrm{Hg}$ were automatically discharged The recording was analyzed to obtain $24 \mathrm{~h}$, daytime and night-time average SBP, DBP and pulse pressure.

The nocturnal pressure drop was calculated according to the following formula:

\section{(\%) $100 \times[1-$ (nocturnal blood pressureldiurnal arterial pressure)]}

The dipper pattern is considered when there is a decrease in the nocturnal AP in relation to the daytime BP greater than $10 \%$, if this decrease is not observed either in the systolic blood pressure or in the diastolic blood pressure the patients are considered non-dippers. 


\section{Statistical analysis}

Values have been expressed as means \pm standard deviation (SD) or as percentages. Comparisons between the two groups relative to quantitative variables were conducted using the t-test for independent samples or the corresponding non-parametric (Mann-Whitney), when the assumption of normality was not verified. The association of independent categorical variables was tested using the $\chi^{2}$ test or Fisher's exact test. In order to determine an independent association of nondipper pattern with posterior wall thickness (PWT) and left ventricular diastolic dysfunction (LVDD) multiple logistic regression analysis adjusting for age, gender was performed All statistical analysis was performed using the statistical software SPSS ${ }^{\otimes}, 25$ for Windows. All tests were two-sided for consistency and $\mathrm{p}<0.05$ was considered to be statistically significant.

\section{Results}

The demographic variables and the ambulatory blood pressure measurement parameters are illustrated in Table I and the echocardiographic and Doppler study parameters in Table II. We enrolled 159 patients, of whom 87 (54.72\%) are males and $72(45.28 \%)$ are females, with a mean age of 43.62 $( \pm 10.73)$ years.

As can be seen in Table I, according to data from the 24-hour ABPM registry of 159 hypertensive patients, 85 patients were classified as dippers and 74 patients as non-dippers. The distribution by age and gender was similar between the two groups $(\mathrm{p}=0.503$ and $\mathrm{p}=0.338$ respectively). As expected, night-time systolic and diastolic pressures were significantly higher in non-dippers than those in dip-

Table I. Demographic and ambulatory blood pressure measurement (ABPM) parameters of studied population

\begin{tabular}{|c|c|c|c|}
\hline \multicolumn{4}{|c|}{ Studied population $(n=159)$} \\
\hline Variables & Non-dipper $(n=74)$ & Dipper $(\mathrm{n}=85)$ & $\mathrm{p}$ value \\
\hline Age (Y) & $44.23 \pm 11.15$ & $43.08 \pm 10.38$ & 0.503 \\
\hline Gender & & & 0.338 \\
\hline Female N, (\%) & $37 \pm 50.0$ & $35 \pm 41.2$ & \\
\hline Male N, (\%) & $37 \pm 50.0$ & $50 \pm 58.8$ & \\
\hline \multicolumn{4}{|l|}{ ABPM } \\
\hline 24-hour SBP [mm Hg] & $135 . .03 \pm 15.21$ & $132.79 \pm 15.30$ & 0.357 \\
\hline 24-hour DBP [mm Hg] & $81.65 \pm 11.66$ & $79.38 \pm 9.38$ & 0.176 \\
\hline Daytime SBP [mm Hg] & $136.72 \pm 15.61$ & $137.69 \pm 16.42$ & 0.702 \\
\hline Daytime DBP [mm Hg] & $83.39 \pm 12.29$ & $83.22 \pm 10.04$ & 0.924 \\
\hline Night-time SBP [mm Hg] & $129.91 \pm 14.63$ & $117.33 \pm 13.38$ & $0.001^{*}$ \\
\hline Nighttime DBP [mm Hg] & $75.30 \pm 11.06$ & $66.86 \pm 9.10$ & $0.001 *$ \\
\hline
\end{tabular}

ABPM — ambulatory blood pressure measurement; SBP — systolic blood pressure; DBP — diastolic blood pressure

Table II. Echocardiographic and Doppler study parameters of studied population

\begin{tabular}{|c|c|c|c|}
\hline \multicolumn{4}{|c|}{ Studied population ( $n=159$ ) } \\
\hline Variables & Non-dipper $(n=74)$ & Dipper $(\mathbf{n}=85)$ & $\mathrm{p}$ value \\
\hline Aortic root [mm] & $32.20 \pm 4.18$ & $31.62 \pm 4.66$ & 0.414 \\
\hline A Ao & $29.68 \pm 4.88$ & $27.8 \pm 5.18$ & $0.001^{*}$ \\
\hline LVDD [mm] & $52.85 \pm 9.24$ & $50.24 \pm 4.16$ & $0.027^{*}$ \\
\hline IVST [mm] & $11.12 \pm 2.03$ & $10.67 \pm 1.90$ & 0.151 \\
\hline PWT [mm] & $10.73 \pm 1.94$ & $10.07 \pm 1.70$ & $0.024^{*}$ \\
\hline $\operatorname{LVEF}(\%)$ & $69.30 \pm 9.29$ & $67.93 \pm 8.46$ & 0.333 \\
\hline $\mathrm{LA}\left[\mathrm{cm}^{2}\right]$ & $19.44 \pm 5.05$ & $19.15 \pm 5.51$ & 0.729 \\
\hline$E / A$ & $1.19 \pm 0.45$ & $1.22 \pm 0.39$ & 0.678 \\
\hline $\mathrm{e}^{\prime}[\mathrm{cm} / \mathrm{s}]$ & $15.60 \pm 5.98$ & $16.01 \pm 5.11$ & 0.647 \\
\hline $\mathrm{E} / \mathrm{e}^{\prime}$ & $5.33 \pm 1.88$ & $5.25 \pm 1.79$ & 0.788 \\
\hline
\end{tabular}

Ao — ascending aorta; LVDD — left ventricular diastolic diameter; IVST — interventricular septal thickness; PWT — posterior wall thickness; LVEF — left ventricular ejection fraction; LA — left atrium 
pers $(129.91 \pm 14.63 \mathrm{~mm} \mathrm{Hg}$ vs. $117.3 \pm 13.38$ $\mathrm{mm} \mathrm{Hg}, \mathrm{p}<0.001)$ and $(75.30 \pm 11.06 \mathrm{~mm} \mathrm{Hg}$ vs. $66.86 \pm 9.10 \mathrm{~mm} \mathrm{Hg}, \mathrm{p}<0.001)$, respectively. The remaining $\mathrm{ABPM}$ parameters were similar in both groups.

The echocardiographic and Doppler study parameters are presented in Table II. Non-dippers had larger ascending aortic diameters $(29.68 \pm 4.88 v$ s. $27.8 \pm 5.18, \mathrm{p}=0.001)$, higher left ventricular diastolic diameters $(52.85 \pm 9.24$ vs. $50.24 \pm 4.16$, $\mathrm{p}=0.027)$ and greater posterior wall thickness $(10.73 \pm 1.94$ vs. $10.07 \pm 1.70, \mathrm{p}=0.024)$ than those in dippers. The dimensions of the aortic root, the thickness of the ventricular septum, as well as left ventricular ejection fraction, likewise, the left atrium area, E/A ratio and E/e ratio were similar in both groups. These data suggest that there are no differences in the LV systolic and diastolic function between dipper and non-dipper hypertensive patients.

On the other hand, the multiple logistic regression analysis adjusted for gender and age revealed that the LVDD $(B=0.103, p=0.001)$ and the posterior wall thickness $(B=0.350, p=0.001)$ are significant estimators of non-dipper hypertensive patients.

\section{Discussion}

In Angola, hypertension has become an increasingly important public health problem [1]. In the study by Pires et al. [1] the prevalence of hypertension in Angola is $23 \%$ (95\% CI: $21 \%$ to $25.2 \%$ ). The authors also noted that among hypertensive individuals, $21.6 \%$ (95\% CI: $17.0 \%$ to $26.9 \%$ ) were aware of their status. Only $13.9 \%$ (95\% CI: $5.9 \%$ to $29.1 \%$ ) of the subjects aware of their condition were under pharmacological treatment, of which approximately one-third were controlled. To our knowledge in Angola there are no studies on the effect of the dipper $v s$. non-dipper pattern on cardiac morphology and function in hypertensive patients.

The main results of the present study revealed that patients with non-dipper pattern have greater left ventricular diastolic diameter and greater posterior wall thickness. In addition, these two parameters proved to be independent predictors of belonging to the non-dipper group. These findings have also been reported by other authors. Yokota et al. [9] report that among hypertensive patients with left ventricular hypertrophy the non-dipper pattern was associated with LV remodeling. Ferrara et al. [10] concluded that the increase in the LV telediastolic dimension was associated with a non-dipper pattern. Verdecchia et al. [11] studied 137 untreated and 98 healthy hypertensive patients concluded that interventricular thickness and posterior wall thickness were associated with the non-dipper pattern. Możdżan et al. compared echocardiographic parameters among hypertensive treated patients with conserved global systolic function concluded that concentric LVH is the prevalent pattern in non-dippers [12]. Ivanovic et al. studied hypertensive patients, most of the sample being untreated for hypertension (69\%), and concluded that structural alterations were associated with the non-dipper and inverted dipper patterns compared with the other patterns [13]. Cuspidi et al. (in 2003) who studied treated - controlled and uncontrolled - hypertensive patients concluded that the non-dipper pattern had no association with increased LV mass and LVH prevalence [5]. But in 2004, when they studied a sample of hypertensive patients diagnosed with dipper $v s$. non-dipper patterns based on two ABPM studies, they concluded that the persistent non-dipper pattern is associated with more cardiac abnormalities [14].

In our study, patients with non-dipper pattern were shown to have larger ascending aorta dimensions. These findings were also reported by Cuspidi et al. [14], who studied recently diagnosed hypertensive patients, and Karaagac et al. [15], who studied 70 hypertensive patients with metabolic syndrome, concluded that the diameter of the thoracic aorta was higher in patients with non-dipper pattern. In turn, Ozdemir et al. [16] verified an association between carotid artery diameter and non-dipper pattern.

In the present study the left atrial size, systolic and diastolic function parameters were similar between the two groups, which is in accordance with results reported by Sokmen et al. [6] and Cuspidi et al. [5] in treated hypertensive patients/patients with controlled hypertension and with the results reported by Grandi et al. [17] in untreated patients. These authors did not find significant differences in left ventricular size, systolic and diastolic function or prevalence of cardiac hypertrophy between the dipper $v$ s. non-dipper patterns. Likewise, Verdecchia et al. [11], found no association between LV systolic function and the dipper pattern. In turn, Della Mea et al. [18], who studied a sample of untreated hypertensive patients, did not find significant differences in diastolic function between dipper and non-dipper patterns.

More recently, in hypertensive patients, 3D echocardiography and strain echocardiography revealed subclinical changes in left ventricular systolic and diastolic function and left atrial function, which have not been demonstrated by conventional echocardiography. These new echocardiographic 
methods become important tools in evaluating the effect of the dipper versus non-dipper patterns on cardiac morphology and function in hypertensive patients [19-23].

A study published in 2016 [19], conducted in newly diagnosed hypertensive patients where cardiac function, in addition to conventional echocardiography, was evaluated by 3D echo, the authors showed that the non-Dipper pattern was associated with $\mathrm{LVH}$ and diastolic dysfunction both by $2 \mathrm{D}$ parameters (E/e increased in the non-Dipper pattern) and by $3 \mathrm{D}$ (increase in the LA minimum volume, the LA pre-contraction volume of the $\mathrm{E}$ and total LA systolic volume).

A study published in 2018 [20], conducted in treated hypertensive patients, where cardiac function beyond conventional echocardiography was also assessed by tissue Doppler and Speckle Tracking with Strain evaluation. In this study, the authors concluded important data on differences in morphology and cardiac function between the dipper vs. non-dipper patterns, demonstrating an association between nondipper pattern and LV remodeling, diastolic dysfunction and reduction of myocardial strain also compatible with LV systolic dysfunction and they alert that patients with uncomplicated hypertension should always consider performing ABPM or another innovative method that provides information on the nocturnal blood pressure pattern to better stratify cardiovascular risk and delineate therapeutic strategies.

The conflicting findings in the existing literature may partially reflect methodological problems [5]. Several factors have been described [5, 17]: first, the inclusion in most of the previous studies of patients who were receiving or had been receiving treatment until shortly before the study may have disturbed in a complex way the relationship between BP and organ damage. In addition, it is possible that the greater prevalence of LVH reported by some studies in non-dippers is a function of a higher cumulative 24-h pressure loads rather than the non-dipping phenomenon per se; second, the definition of the night-time and daytime periods. Some authors have defined night-time BP somewhat arbitrarily as the BP during the hours most likely to correspond to sleep, without referring to diaries reporting when patients actually retire and awake. In contrast, others have used narrower time spans that exclude the morning and evening transitional periods during which a variable proportion of patients are actually awake or asleep. Finally, the classification into dippers and non-dippers has a low reproducibility over time; this limited reproducibility is presumably related to the fact that the quality and depth of sleep as well as the mental and physical activity during daytime can markedly vary from one recording session to another.

In the present study, we compared left ventricular function and aortic dimensions between dipper and non-dipper patterns in hypertensive patients with more than one year of evolution. The definition of dippers and non-dippers was based in only one 24-hour ABPM study, with fixed definition of the daytime and nigh-time periods. Using this methodology we found more alterations in left ventricular morphology and ascending aortic diameter in non-dipper patients. No differences regarding left ventricular systolic and diastolic function between groups were found.

\section{Limitations of the study}

The present study has a number of limitations that we would like to emphasize. Although the population studied had been diagnosed with hypertension for over a year, we do not know if everyone was taking medication. The evaluation of cardiac function was performed using conventional echocardiography, Doppler study and tissue Doppler, but more recent techniques such as 3D echocardiography or strain were not used. Finally, we did not evaluate the presence of other cardiovascular risk factors that could have better characterized the study population.

\section{Conclusion}

The results show that hypertensive patients with more than one year of evolution presented with nondipper pattern have more alterations in left ventricular geometry, but not in left ventricular systolic and diastolic function. We believe that further studies on the effect of dipper and non-dipper patterns on hypertensive patients in Africa are needed to confirm the data presented here.

None to declare.

\section{Conflict of interests}

None to declare.

\section{Acknowledgments}

Financial support

None.

\section{References}

1. Pires JE, Sebastiáo YV, Langa AJ, et al. Hypertension in Northern Angola: prevalence, associated factors, awareness, treatment and control. BMC Public Health. 2013; 13: 90, doi: 10.1186/14712458-13-90, indexed in Pubmed: 23363805. 
2. Marwick TH, Gillebert TC, Aurigemma G, et al. Recommendations on the Use of Echocardiography in Adult Hypertension: A Report from the European Association of Cardiovascular Imaging (EACVI) and the American Society of Echocardiography (ASE). Eur Heart J Cardiovasc Imaging. 2015; 16(6): 577-605, doi: 10.1093/ehjci/ jev076, indexed in Pubmed: 25995329.

3. Madin K, Iqbal P. Twenty four hour ambulatory blood pressure monitoring: a new tool for determining cardiovascular prognosis. Postgrad Med J. 2006; 82(971): 548-551, doi: 10.1136/pgmj.2006.046409, indexed in Pubmed: 16954448.

4. Parati G, Stergiou G, O’Brien E, et al. European Society of Hypertension Working Group on Blood Pressure Monitoring and Cardiovascular Variability, European Society of Hypertension Working Group on Blood Pressure Monitoring. European Society of Hypertension position paper on ambulatory blood pressure monitoring. J Hypertens. 2013; 31(9): 1731-1768, doi: 10.1097/HJH.0b013e328363e964, indexed in Pubmed: 24029863.

5. Cuspidi C, Michev I, Meani S, et al. Non-dipper treated hypertensive patients do not have increased cardiac structural alterations. Cardiovascular Ultrasound. 2003; 1(1), doi: 10.1186/1476-7120-1-1.

6. Sokmen G, Sokmen A, Aksu E, et al. The influence of ambulatory blood pressure profile on global and regional functions of the left and the right ventricles in orderly treated hypertensive patients. Echocardiography. 2008; 25(5): 465-472, doi: 10.1111/j.15408175.2008.00632.x, indexed in Pubmed: 18279401.

7. Nagueh SF, Appleton CP, Gillebert TC, et al. Recommendations for the evaluation of left ventricular diastolic function by echocardiography. J Am Soc Echocardiogr. 2009; 22(2): 107-133, doi: 10.1016/j. echo.2008.11.023, indexed in Pubmed: 19187853.

8. Quiñones M, Otto C, Stoddard M, et al. Recommendations for quantification of Doppler echocardiography: A report from the Doppler quantification task force of the nomenclature and standards committee of the American Society of Echocardiography. J Am Soc Echocardiogr. 2002; 15(2): 167-184, doi: 10.1067/ mje.2002.120202, indexed in Pubmed: 11836492.

9. Yokota H, Imai Y, Tsuboko Y, et al. Nocturnal Blood Pressure Pattern Affects Left Ventricular Remodeling and Late Gadolinium Enhancement in Patients with Hypertension and Left Ventricular Hypertrophy. PLoS One. 2013; 8(6): e67825, doi: 10.1371/journal. pone.0067825, indexed in Pubmed: 23840777.

10. Ferrara LA, Guida L, Mangini FP, et al. Cardiovascular abnormalities in never-treated hypertensives according to nondipper status. Am J Hypertens. 1998; 11(11 Pt 1): 1352-1357, doi: 10.1016/s08957061(98)00164-2, indexed in Pubmed: 9832179.

11. Verdecchia P, Schillaci G, Borgioni C, et al. Circadian blood pressure changes and left ventricular hypertrophy in essential hypertension. Circulation. 1990; 81(2): 528-536, doi: 10.1161/01.cir.81.2.528, indexed in Pubmed: 2137047.

12. Możdżan M, Wierzbowska-Drabik K, Kurpesa M, et al. Echocardiographic indices of left ventricular hypertrophy and diastolic function in hypertensive patients with preserved LVEF classified as dippers and non-dippers. Arch Med Sci. 2013; 9(2): 268-275, doi: 10.5114/ aoms.2013.34534, indexed in Pubmed: 23671437.

13. Ivanovic BA, Tadic MV, Celic VP. To dip or not to dip? The unique relationship between different blood pressure patterns and cardiac function and structure. J Hum Hypertens. 2013; 27(1): 62-70, doi: 10.1038/jhh.2011.83, indexed in Pubmed: 21900953.

14. Cuspidi C, Meani S, Salerno M, et al. Cardiovascular target organ damage in essential hypertensives with or without reproducible nocturnal fall in blood pressure. J Hypertens. 2004; 22(2): 273-80, doi: 10.1097/00004872-200402000-00010, indexed in Pubmed: 15076184.

15. Karaagac K, Vatansever F, Tenekecioglu E, et al. The Relationship between Non-Dipper Blood Pressure and Thoracic Aortic Diameter in Metabolic Syndrome. Eurasian J Med. 2014; 46(2): 120-125, doi: 10.5152/eajm.2014.27, indexed in Pubmed: 25610310.

16. Ozdemir E, Yildirimturk O, Cengiz B, et al. Evaluation of carotid intima-media thickness and aortic elasticity in patients with nondipper hypertension. Echocardiography. 2014; 31(5): 663-668, doi: 10.1111/echo.12444, indexed in Pubmed: 24219389.

17. Grandi AM, Broggi R, Jessula A, et al. Relation of extent of nocturnal blood pressure decrease to cardiovascular remodeling in never-treated patients with essential hypertension. Am J Cardiol. 2002; 89(10): 1193-1196, doi: 10.1016/s0002-9149(02)02303-2, indexed in Pubmed: 12008174.

18. Della Mea P, Lupia M, Bandolin V, et al. Adiponectin, insulin resistance, and left ventricular structure in dipper and nondipper essential hypertensive patients. Am J Hypertens. 2005; 18(1): 30-35, doi: 10.1016/j.amjhyper.2004.08.029, indexed in Pubmed: 15691614.

19. Ermiş N, Otlu YÖ, Afşin A, et al. Comparison of left atrial volume and function in non-dipper versus dipper hypertensives: A real-time three-dimensional echocardiography study. Anatol J Cardiol. 2016; 16(6): 428-433, doi: 10.14744/AnatolJCardiol.2015.6569, indexed in Pubmed: 27182617.

20. Chen Y, Liu JH, Zhen Z, et al. Assessment of left ventricular function and peripheral vascular arterial stiffness in patients with dipper and non-dipper hypertension. J Investig Med. 2018; 66(2): 319-324, doi: 10.1136/jim-2017-000513, indexed in Pubmed: 28935634.

21. Park CS, An GH, Kim YW, et al. Evaluation of the Relationship between Circadian Blood Pressure Variation and Left Atrial Function Using Strain Imaging. J Cardiovasc Ultrasound. 2011; 19(4): 183-191, doi: 10.4250/jcu.2011.19.4.183, indexed in Pubmed: 22259661.

22. Açar G, Bulut M, Arslan K, et al. Comparison of left atrial mechanical function in nondipper versus dipper hypertensive patients: a speckle tracking study. Echocardiography. 2013; 30(2): 164-170, doi: 10.1111/echo.12023, indexed in Pubmed: 23167459.

23. Tadic M, Cuspidi C, Celic V, et al. Nocturnal hypertension and right heart remodeling. J Hypertens. 2018; 36(1): 136-142, doi: $10.1097 /$ HJH.0000000000001506, indexed in Pubmed: 28817494. 